\title{
Expressão gênica de ApoA1 e ApoB em frangos de corte de duas linhagens diferentes
}

[Genic expression of ApoAl and ApoB in liver of male broilers from different strains]

\section{"Comunicação Breve/Short Communication"}

\author{
Angélica de Souza Khatlab ${ }^{1}$, Stefânia Caroline Claudino da Silva ${ }^{2}$, André Luiz Seccatto Garcia ${ }^{3}$, \\ Éverton Ferreira da Silva ${ }^{1}$, Marcelise Fachinello ${ }^{1}$, Eliane Gasparino ${ }^{1}$ \\ ${ }^{1}$ Universidade Estadual de Maringá, Maringá-PR, Brasil \\ ${ }^{2}$ Centro Universitário de Maringá, Maringá-PR, Brasil. \\ ${ }^{3}$ University of Georgia, Athens, Georgia, United States of America. \\ *Autor para correspondência/Corresponding author: E-mail: stefania.silva@ unicesumar.edu.br
}

\section{Resumo}

O objetivo desse estudo foi avaliar a expressão gênica das apolipoproteínas A1 e B, bem como a relação entre elas no fígado de frangos de corte de duas linhagens, selecionada e não selecionada. O experimento foi conduzido na fazenda experimental da Universidade Estadual de Maringá. Os animais foram alojados em gaiolas em ambiente climatizado durante 42 dias. As dietas oferecidas foram adequadas a cada fase de desenvolvimento, e não diferiram entre os dois grupos. Ao final desse período os animais foram abatidos e foram realizadas coletas de fígado para as análises de expressão gênica. As amostras foram armazenadas em tubo criogênico imerso em nitrogênio líquido até o momento da extração de RNA das amostras. Os animais da linhagem selecionada apresentaram menor expressão relativa dos genes estudados, quando comparados com os animais da linhagem não selecionada, entretanto, a relação entre as apolipoproteínas se manteve constante nas duas linhagens. A seleção para ganho de peso reduz a expressão dos genes das apolipoproteínas A1 e B no fígado de frangos de corte.

Palavras-chave: apolipoproteínas; Gallus gallus; RT-PCR.

\begin{abstract}
The aim of this study was to evaluate the gene expression of apolipoproteins A1 and B, as well as the relationship between them in the liver from broilers of two strains: selected and not selected. The experiment was conducted at the experimental farm of the State University of Maringá and the animals were housed in bioclimatic cages for 42 days. The diet offered was adequate at each stage of development and did not differ between the two groups. At the end of the experiment, the animals were slaughtered, and liver samples were collected for analysis of gene expression. Samples were stored in a cryogenic tube immersed in liquid nitrogen until the extraction of RNA. Animals of the selected strain showed lower relative expression of the studied genes when compared to the animals of the unselected strain. However, the ratio between apolipoproteins remained constant in both strains. Selection for weight gain reduces expression of apolipoprotein A1 and B genes in broiler liver.
\end{abstract}

Keywords: apolipoproteins; Gallus gallus; RT-PCR.

As lipoproteínas são complexos hidrofílicos esféricos, que transportam lipídios hidrofóbicos em seu núcleo. Estes complexos são formados por proteínas, chamadas de apoproteínas ou apolipoproteínas (Apo), além de colesterol, ésteres de colesterol, triglicerídeos e fosfolipídios. A presença das Apos no complexo lipoproteico, confere a este o caráter hidrofílico, tornando-o solúvel no plasma, além de promover a captação dos lipídios pelos tecidos (Beisiegel, 1998). As Apos são divididas em vários grupos, dentre eles: ApoA, ApoB, ApoC, ApoE e Apo(a), com destaque para ApoA1 e B, por estarem relacionadas com risco cardiovascular (Barter e Kastelein, 2006). 
O gene que codifica para a apolipoproteína A1 (ApoA1) em aves é expresso em todos os tecidos (Shackelford e Lebherz, 1983; Lamon-Fava et al., 1992), sendo o fígado e o intestino, os principais locais de síntese do mRNA (LamonFava et al., 1992). Nos tecidos, a ApoA1 atua como coenzima para a enzima lecitina colesterolaciltransferasee também como um mediador na transferência do colesterol das células para as partículas de lipoproteínas de alta densidade (HDL), atuando como ligante para o ABCA-I (transportador que promove o fluxo de colesterol livre e fosfolipídeos das células) (Walldius e Jungner, 2004; Forti e Diament, 2006).

A ApoB é sintetizada no fígado (Richardson et al., 2005), e em aves é o principal componente de IDL (Lipoproteína de densidade intermediária) e VLDL plasmático (Hermier et al., 1985), sendo responsável pela ligação das partículas de LDL aos receptores celulares, permitindo a entrada de LDL nas células. Neste contexto, um excesso de ApoB pode representar um fator desencadeante para $o$ processo aterogênico (Denke, 2005; Barter e Kastelein, 2006). Polimorfismos no gene da ApoB foram associados à melhores taxas de crescimento iniciais e menor deposição de gordura corporal (Zhang et al., 2006). De forma semelhante, Da Cruz et al. (2015) demonstraram a existência de associação significativa entre polimorfismos no gene ApoB, à baixa deposição de gordura abdominal e subcutânea, bem como à distúrbios esqueléticos em frangos de corte.

Considerando o papel das AposA1 e B no transporte dos lipídeos, e as evidencias de associação genética com melhores taxas de crescimento e baixa deposição de gordura corporal (Seyedabadi et al., 2010), estudos sobre a expressão destes genes em aves de diferentes linhagens podem auxiliar em futuros programas de seleção e melhoramento genético. Deste modo, objetivamos avaliar a expressão gênica das ApoA1, ApoB e sua relação no fígado de duas linhagens de frango de corte, selecionadas e não selecionadas.

Foram utilizados 20 frangos de corte (Gallus gallus) machos de linhagem comercial selecionada (Cobb) e 20 frangos de corte machos de linhagem não selecionada, alocados na Fazenda experimental da Universidade Estadual de Maringá, na cidade de Iguatemi- PR.

Os animais das duas linhagens foram alojados em gaiolas em sala climatizada e submetidos às mesmas condições ambientais, sendo alimentados com a mesma dieta e plano alimentar, de acordo com a fase de criação. Durante o período experimental, os animais tiveram livre acesso à água e ração balanceada baseada em milho e soja (Rostagno et al., 2011). Na fase inicial foi fornecida ração com $21,60 \%$ de proteína bruta e $3052 \mathrm{Kcal} / \mathrm{Kg}$ de energia, enquanto na fase de crescimento foi fornecida ração contendo $19,70 \%$ de proteína bruta e $3170 \mathrm{Kcal} / \mathrm{Kg}$ de energia.

Aos 42 dias de idade, quatro animais de cada linhagem foram submetidos a um período de 6 horas de jejum pré-abate e abatidos por método de deslocamento cervical. Foram coletadas amostras de fígado e armazenadas em tubo criogênico imerso em nitrogênio líquido até o momento da extração do RNA. Antes da extração do RNA das amostras, todos os materiais utilizados foram tratados com inibidor de RNAse AWAY $^{\circledR}$ (Invitrogen, Carlsbad, CA, EUA).

Para extração do RNA, foi utilizado reagente Trizol $^{\circledR}$ (Invitrogen, Carlsbad, CA, EUA), nas proporções de $100 \mathrm{mg}$ de tecido por $1 \mathrm{~mL}$ do produto, de acordo com o protocolo indicado pelo fabricante.

O RNA total foi avaliado utilizando espectrofotômetro Nanodrop 2000 UV-Vis (ThermoScientific, Waltham, MA, EUA), por meio da relação entre os comprimentos de onda de 260/280 nm. A integridade do RNA foi analisada usando gel de agarose $1 \%$ marcado com Sybr Safe ${ }^{\circledR}$ DNA Gel Stain (Invitrogen, Carlsbad, CA, EUA), com leitura realizada sob luz ultravioleta. Após avaliação da integridade do RNA total, as amostras foram tratadas com DNase I, Amplification Grade (Invitrogen, Carlsbad, CA, EUA) utilizando os procedimentos indicados pelo fabricante, para evitar possíveis contaminações com DNA genômico. Para a síntese de DNA, foi utilizado kit SuperScript ${ }^{\circledR}$ III First-Strand Synthesis Super Mix (Invitrogen, Carlsbad, CA, EUA), de acordo com os protocolos indicado pelo fabricante. $\mathrm{O}$ cDNA sintetizado foi armazenado em freezer a $-20{ }^{\circ} \mathrm{C}$.

Os primers foram desenhados com base nas sequências de genes depositadas no banco de dados disponível em www.ncbi.nlm.nih.gov (códigos de referências M25559.1, NM_001044633.1e L08165.1, respectivamente para os genes ApoA1, ApoB e CHKBACTN), utilizando a ferramenta do site www.idtdna.com (Tabela 1). 
Tabela 1. Sequências dos primers utilizados para reação de PCR em tempo real.

\begin{tabular}{lccl}
\hline & amplicon $(\mathbf{p b})$ & temperatura de anelamento $\left({ }^{\circ} \mathbf{C}\right)$ & \multicolumn{1}{c}{ sequência do primer $\left(\mathbf{5}^{\prime}-\mathbf{3}^{\prime}\right)$} \\
\hline apoA1 & 214 & 60,0 & GTGACCCTCGCTGTGCTCTT \\
& & & CACTCAGCGTGTCCAGGTTGT \\
apoB & 196 & 60,0 & GACTTGGTTACACGCCTCA \\
& & & TAACTTGCCTGTTATGCTC \\
CHKBACTN & 136 & 60,0 & ACCCCAAAGCCAACAGA \\
& & & CCAGAGTCCATCACAATACC
\end{tabular}

Para as reações de RT-PCR, foi utilizado o corante fluorescente SYBR ${ }^{\circledR}$ Green PCR Master Mix (Applied Biosystems ${ }^{\mathrm{TM}}$, Carlsbad, CA, EUA), e as reações processadas em Step One Plus ${ }^{T M}$ RealTime PCR System, Applied Biosystems ${ }^{\mathrm{TM}}$, Carlsbad, CA, EUA. Todas as reações foram feitas em duplicata, utilizando um volume total de $25 \mu \mathrm{L}$ por reação.

A relação entre CT (Treshold Cicle) do gene em e CT do controle endógeno foi corrigida, considerando os valores de $2^{-\Delta c t}$, sendo $\Delta \mathrm{ct}=\mathrm{CT}$ do gene alvo - CT do controle endógeno. Para a análise estatística dos dados, foi utilizado o pacote estatístico SAS (SAS Inst. Inc., Cary, NC, EUA), utilizando teste de médias de Tukey $(\mathrm{P}<0,05)$. Os resultados foram mostrados como média e erro padrão.

No teste de eficiência dos primers, os genes estudados apresentaram variação entre 90 e 100\% de eficiência. A análise das curvas de dissociação confirmou a especificidade das reações e a ausência da formação de dímeros de primers. $\mathrm{O}$ gene CHKBACTIN foi utilizado como controle endógeno devido à elevada eficiência de sua amplificação, e por não apresentar variação estatística significativa entre os tratamentos.

Os valores de expressão relativa dos genes (ApoA1 e ApoB) são mostrados na Tabela 2. Houve diferença significativa entre a expressão relativa dos genes em ambas as linhagens utilizadas. Os machos da linhagem não selecionada para ganho de peso apresentaram maior nível de expressão gênica tanto para ApoA1 quanto para ApoB.

A correlação entre a expressão gênica de ApoA1 e ApoB foi alta $(\mathrm{r}=0,81 ; \mathrm{P}<0,001)$ demonstrando forte associação entre as apoproteínas.

Nesse estudo, observou-se que os animais da linhagem selecionada para ganho de peso apresentaram menor expressão relativa do gene ApoA1 (Tabela 2). As ApoA1 são o principal constituinte proteico do HDL, molécula responsável por facilitar a remoção do colesterol dos tecidos periféricos levando-o ao fígado. A ApoA1 atua neste processo como coenzima para aciltransferase lecitina-colesterol (LCAT), a principal enzima responsável por catalisar a maturação de HDL nascente em HDL esférico, promovendo a esterificação do colesterol livre (Fielding et al., 1972). Devido ao seu papel no transporte reverso do colesterol, níveis reduzidos de HDL no plasma têm sido associados com maior propensão de formação de placas aterogênicas (Leança et al., 2010).

Em frangos, a supressão da expressão da ApoA1 pode afetar a formação da HDL, prejudicando desse modo o processo de transporte reverso de colesterol, resultado em maior acúmulo de gordura abdominal, como sugerido por Zhuo et al. (2015). Os níveis de expressão de ApoA1 podem oscilar durante a vida dos animais. Em frangos, por exemplo, é possível observar aumento dos níveis de ApoA1 próximo ao período de eclosão, seguido de queda brusca de sua expressão após esse período (Shackelford e Lebherz, 1983). Estes mesmos autores relataram aumento significativo da expressão gênica de ApoA1 no músculo esquelético distrófico de aves adultas, entretanto, este aumento não foi observado em aves de dois dias de idade, sugerindo provável relação da expressão de ApoA1 com a idade, e consequentemente, com o teor de gordura corporal (Shackelford e Lebherz, 1985).

Daval et al. (2000) avaliaram a expressão de ApoA1 em linhagens de frangos de corte adultos (56 dias) divergentes quanto a deposição de gordura abdominal. Estes autores observaram que a linhagem de frangos magros apresentaram menor expressão de ApoA1, bem como menores níveis séricos desta quando comparados com animais de linhagem gorda (transcritos de RNAm: 24,212 unidades arbitrárias (UA) para frangos gordos e 10,685 UA para frangos magros). Estes autores encontraram em frangos de corte adulto, expressão de ApoA1 aproximadamente 2,3 vezes maior em 
frangos gordos quando comparados com frangos magros.

A redução de ApoA1 observada para aves selecionadas neste trabalho sugerem que, o processo de melhoramento genético pelo qual a linhagem foi submetida tenha indiretamente selecionado aves menos eficientes em expressar ApoA1, provavelmente, por desequilíbrio de ligação com alelos de características de crescimento. Apesar da menor expressão da ApoA1 nos animais selecionados, estudos tem sugerido que os níveis lipídicos da carcaça não podem ser avaliados com base apenas nos níveis desta lipoproteína, e sim da relação entre proteínas de síntese direta e reversa de colesterol. Neste contexto, a avaliação de ApoA1 e ApoB representam uma medida mais acurada para esta avaliação.

Semelhantemente à ApoA1, observamos neste estudo redução significativa da expressão gênica de ApoB para machos selecionados (Tabela 2).

Tabela 2. Expressão dos genes ApoA1 e ApoB em 304 amostras de fígado de frangos de corte machos da linhagem Cobb e frangos de corte não selecionados para ganho em peso.

\begin{tabular}{llll}
\hline & apoA1 & apoB & apoB/apoA1 \\
\hline & média $\pm \mathrm{ep}^{1}$ & média $\pm \mathrm{ep}^{1}$ & \\
$\mathrm{~ms}^{2}$ & $3,65 \pm 0,46^{*}$ & $0,26 \pm 0,03^{*}$ & $0,072 \pm 0,008^{\text {ns }}$ \\
$\mathrm{mns}^{3}$ & $8,93 \pm 0,74^{*}$ & $0,57 \pm 0,08^{*}$ & $0,064 \pm 0,012^{\text {ns }}$ \\
$P$ & $<0,0001$ & 0,0038 & \\
\hline
\end{tabular}

* diferença significativa $(\mathrm{P}<0,05)$

1 ep $=$ erro padrão

${ }^{2} \mathrm{~ms}=$ Machos selecionados

${ }^{3} \mathrm{mns}=$ Machos não selecionados

Altas concentrações de ApoB no plasma são frequentemente relacionadas à doenças cardiovasculares, devido a sua função no transporte direto do colesterol circulante para tecidos periféricos, por meio de duas formas distintas: apoB100 e apoB48. A apoB100 é o componente proteico das LDL, quilomícrons e VLDL, enquanto apoB48 é encontrada apenas nos quilomícrons(Walldius e Jungner, 2006). A relação entre maiores índices de ApoB, potencial aterogênico e provável obesidade foi primariamente relatada por Kissebah et al., (1981) e revisada por Lemieux (1997). Em frangos de corte, esta relação foi confirmada por Zhang et al. (2006) por meio de associação de índices de obesidade à polimorfismos genéticos.

A expressão gênica de $A p o B$ no saco vitelínico de embriões de frango está relacionada à idade do embrião, e é expresso de maneira coordenada com a proteína de transferência de triglicerídeo microsomal (MTP), responsável pela transferência dos triglicerídeos para a ApoB, permitindo a formação da VLDL (Eresheim et al., 2014). A menor expressão relativa para $A$ poB observada nas aves selecionadas pode ser decorrente da seleção direta para maior deposição de carne magra, e consequentemente, menor taxa de deposição de gordura nos tecidos. A menor expressão gênica de ApoA1 e ApoB para a linhagem selecionada sugerem que estas aves possuem quantitativamente menores índices de colesterol circulante, sejam HDL ou não HDL, entretanto, tais dosagens não foram realizadas neste experimento.

A relação entre ApoB/ApoA1 é importante para o adequado transporte lipídico (Barter e Kastelein, 2006), dado que ApoA1 está presente em partículas potencialmente antiaterogênicas e ApoB em partículas aterogênicas. Sato et al. (2016) avaliaram a correlação entre ApoA1, ApoB e potencial aterogênico $\mathrm{e}$ antiaterogênico e observaram que Apo A-1 foi significativamente correlacionado com HDL $(r=0,86)$ e apo B foi significativamente correlacionada com LDL, colesterol não HDL e a relação LDL / HDL ( $\mathrm{r}=$ $0,86,0,93$ e 0,64 , respectivamente) evidenciando a necessidade da manutenção das relações adequadas entre ApoB e ApoA1. O valor numérico da relação ApoB/ApoA1 varia de acordo com a espécie (Tabela 3).

Neste trabalho, não foram observadas diferenças significativas entre a relação ApoB/ApoA1 para as linhagens estudadas. Estes dados sugerem que apesar da redução significativa da expressão de ApoB e ApoA1 em frangos selecionados, tal redução não interfere na capacidade cardiovascular nem na qualidade lipídica das carcaças uma vez que a relação ApoB/ApoA1 foi mantida. 
Tabela 3. Relação ApoB/ApoA1em três espécies distintas.

\begin{tabular}{|c|c|c|c|c|}
\hline Espécie & $\begin{array}{c}\text { ApoB/ApoA1 } \\
\text { (mRNA) }\end{array}$ & $\begin{array}{c}\text { ApoB/ApoA1 } \\
\text { (sérico) }\end{array}$ & Artigo & Autor \\
\hline Humanos & - & $0,76 \pm 0,09$ & $\begin{array}{l}\text { Observed relationship between } \\
\text { ratios HDL-cholesterol/total } \\
\text { cholesterol and apolipoprotein } \\
\text { A1/apolipoprotein B }\end{array}$ & $\begin{array}{l}\text { Kuyl e } \\
\text { Mendelsohn } \\
\text { (1992) }\end{array}$ \\
\hline Frangos & - & $0,19 \pm 0,03$ & $\begin{array}{l}\text { The combined effects of novel } \\
\text { tocotrienols and lovastatin on lipid } \\
\text { metabolism in chickens }\end{array}$ & $\begin{array}{c}\text { Qureshi e } \\
\text { Peterson (2001) }\end{array}$ \\
\hline Peixes & $0,97 \pm 0,04$ & - & $\begin{array}{l}\text { Dietary Lipid and Carbohydrate } \\
\text { Interactions: Implications } \\
\text { on Lipid and Glucose Absorption, } \\
\text { Transport in Gilthead Sea } \\
\text { Bream (Sparus aurata) Juveniles }\end{array}$ & $\begin{array}{l}\text { Castro et al. } \\
\text { (2016) }\end{array}$ \\
\hline
\end{tabular}

A seleção direta de aves para características de ganho em peso reduziu a expressão gênica das apoproteínas A1 e B nas aves avaliadas. Apesar da redução significativa da expressão individual das apoproteínas A1 e B para aves selecionadas, não há comprometimento da capacidade de balanço de transporte lipídico, uma vez que a relação entre estas é mantida.

\section{Conflito de Interesse}

Os autores declaram não existir conflito de interesse.

\section{Comitê de Ética}

Este experimento foi aprovado pelo comitê ético para uso de animais em experimentação da Universidade Estadual de Maringá (CEUA $\mathrm{n}^{\circ}$.4000170615).

\section{Referências}

Barter, P.J.; Kastelein, J.J.P. Targeting cholesteryl ester transfer protein for the prevention and management of cardiovascular disease. Journal of the American College of Cardiology, 47(3): 492-499, 2006.

Beisiegel, U. Lipoprotein metabolism. European Heart Journal, 19(A):20-23, 1998.

Castro, C., Corraze, G., Basto, A., Larroquet, L., Panserat, S., Oliva-Teles, A. Dietary lipid and carbohydrate interactions: implications on lipid and glucose absorption, transport in gilthead sea bream (Sparus aurata) juveniles. Lipids, 51(6): 743-755, 2016.

Da Cruz, V.A.R.; Schenkel, F.S.; Savegnago, R.P.; Grupioni, N.V.; Stafuzza, N.B.; Sargolzaei, M.; Ibelli, A.M.G.; Peixoto, J.O.; Ledur,
M.C.; Munari, D.P. Association of apolipoprotein B and adiponectin receptor 1 genes with carcass, bone integrity and performance traits in a paternal broiler line. PLOS ONE, 10(8): 1-16, 2015.

Daval, S.; Lagarrigue, S.; Douaire, M. Messenger RNA levels and transcription rates of hepatic lipogenesis genes in genetically lean and fat chickens. Genetics Selection Evolution, 32: 521-531, 2000.

Denke, M.A. Weighing in before the fight: lowdensity lipoprotein cholesterol and non-highdensity lipoprotein cholesterol versus apolipoprotein B as the best predictor for coronary heart disease and the best measure of therapy. Circulation, 112(22): 3368-3370, 2005.

Eresheim, C.; Plieschnig, J.; Ivessa, N.E.; Schneider, W.J.; Hermann, M. Expression of microsomal triglyceride transfer protein in lipoprotein-synthesizing tissues of the developing chicken embryo. Biochimie, 101(1): 67-74, 2014.

Fielding, C.J.; Shore, V.G.; Fielding, P.E. A protein cofactor of lecithin: cholesterol acyltransferase. Biochemical and Biophysical Research Communications, 46(4): 1493-1498, 1972.

Forti, N.; Diament, J. Lipoproteínas de alta densidade: aspectos metabólicos, clínicos, epidemiológicos e de intervenção terapêutica. Atualização para os clínicos. Arquivos Brasileiros de Cardiologia, 87(5): 671-679, 2006.

Hermier, D.; Forgez, P.; John, C.M. A density gradient study of the lipoprotein and 
apolipoprotein distribution in the chicken, Gallus domesticus. Biochimica et Biophysica Acta, 836(1): 105-118, 1985.

Kissebah, A.H.; Alfarsi, S.; Adams, P.W. Integrated regulation of very low density lipoprotein triglyceride and apolipoprotein-B kinetics in man: normolipemic subjects, familial hypertriglyceridemia and familial combined hyperlipidemia. Metabolism, 30(9): 856-868, 1981.

Kuyl, J.M.; Mendelsohn, D. Observed relationship between ratios HDL-cholesterol/total cholesterol and apolipoprotein A1/apolipoprotein B. Clinical Biochemistry, 25(5): 313-316, 1992.

Lamon-Fava, S.; Sastry, R.; Ferrari, S.; Rajavashisth, T.B.; Lusis, A.J.; Karathanasis, S.K. Evolutionary distinct mechanisms regulate apolipoprotein A-I gene expression: differences between avian and mammalian apoA-I gene transcription control regions. Journal of Lipid Research, 33(6): 831-842, 1992.

Lemieux, S. Genetic susceptibility to visceral obesity and related clinical implications. International journal of obesity and related metabolic disorders: journal of the International Association for the Study of Obesity, 21(10): 831-838, 1997.

Qureshi, A.A.; Peterson, D.M. The combined effects of novel tocotrienols and lovastatin on lipid metabolism in chickens. Atherosclerosis, 156(1): 39-47, 2001.

Richardson, P.E.; Manchekar, M.; Dashti, N.; Jones, M.K.; Beigneux, A.; Young, S.G.; Harvey, S.C.; Segrest, J.P. Assembly of lipoprotein particles containing apolipoprotein-B: structural model for the nascent lipoprotein particle. Biophysical Journal, 88(4): 2789-2800, 2005.
Sato, Y.; Fujimoto, S.; Toida, T.; Nakagawa, H. ; Yamashita, Y.; Iwakiri, T.; Fukuda, A.; Iwatsubo, S. Apoprotein B/apoprotein A-1 ratio and mortality among prevalent dialysis patients. Clinical Journal of the American Society of Nephrology, 11(5): 840-846, 2016.

Saeedi, R.; Li, M.; Frohlich, J. A review on lecithin: cholesterol acyltransferase deficiency. Clinical Biochemistry, 48(7-8): 472-475, 2015.

Seyedabadi, H.R.; Amirinia, C.; Amirmozafari, N.; Torshizi, R.V.; Chamani, M.; Aliabad, A.J. Association of apolipoprotein B gene with body growth and fatness traits in Iranian commercial broiler lines. Livestock Science, 132(1-3):177-181, 2010.

Shackelford, J.E.; Lebherz, H.G. Regulation of apolipoprotein A1 synthesis in avian muscles. Journal of Biological Chemistry, 258(24): 14829-14833, 1983.

Shackelford, J.E.; Lebherz, H.G. Synthesis of apolipoprotein A1 in skeletal muscles of normal and dystrophic chickens. Journal of Biological Chemistry, 260(1): 288-291, 1985.

Walldius, G.; Jungner, I. Apolipoprotein B and apolipoprotein A-I: risk indicators of coronary heart disease and targets for lipid-modifying therapy. Journal of Internal Medicine, 255(2): 188-205, 2004.

Walldius, G.; Jungner, I. The apoB/apoA-I ratio: a strong, new risk factor for cardiovascular disease and a target for lipid-lowering therapy - A review of the evidence. Journal of Internal Medicine, 259(5): 493-519, 2006.

Zhang, S.; Li, H.; Shi, H. Single marker and haplotype analysis of the chicken apolipoprotein B gene T123G and D9500D9polymorphism reveals association with body growth and obesity. Poultry Science, 85(2): 178-84, 2006. 